This item was submitted to Loughborough's Research Repository by the author.

Items in Figshare are protected by copyright, with all rights reserved, unless otherwise indicated.

\title{
Dual coupling networks
}

PLEASE CITE THE PUBLISHED VERSION

http://dx.doi.org/10.1243/09544062C09405

PUBLISHER

Professional Engineering Publishing / @ IMechE

VERSION

VoR (Version of Record)

LICENCE

CC BY-NC-ND 4.0

REPOSITORY RECORD

Davies, T.H.. 2019. "Dual Coupling Networks". figshare. https://hdl.handle.net/2134/4667. 
This item was submitted to Loughborough's Institutional Repository (https://dspace.lboro.ac.uk/) by the author and is made available under the following Creative Commons Licence conditions.

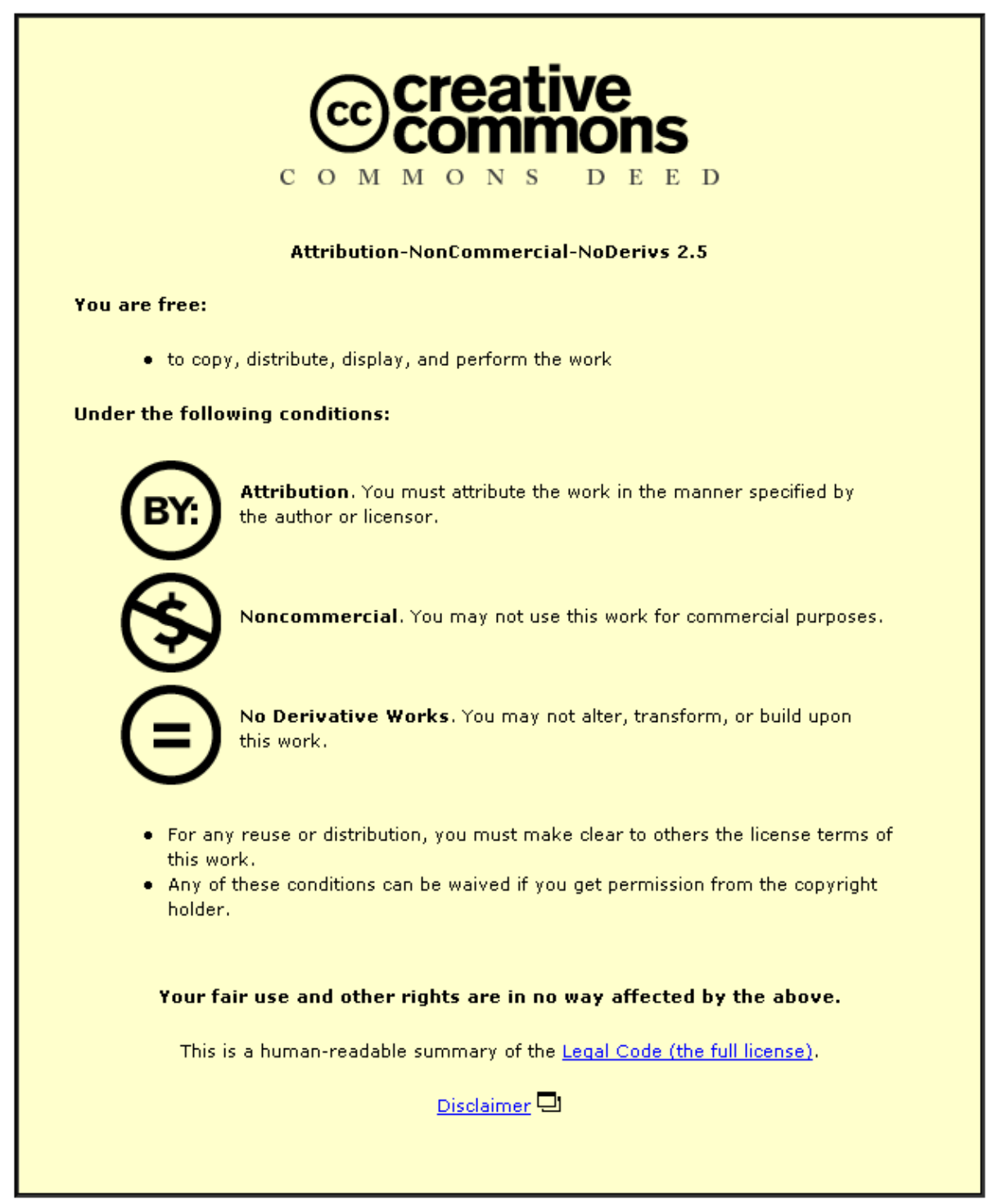

For the full text of this licence, please go to: http://creativecommons.org/licenses/by-nc-nd/2.5/ 


\title{
Dual coupling networks
}

T H Davies

Wolfson School of Mechanical and Manufacturing Engineering, Loughborough University, Loughborough, Leicestershire LE11 3TU, UK

The manuscript was received on 13 November 2005 and was accepted after revision for publication on 13 March 2006.

DOI: $10.1243 / 09544062 C 09405$

\begin{abstract}
Using dual graphs and dual couplings, it is shown how the dual of a coupling network can be found. The actions that can exist within an overconstrained coupling network are shown to be geometrically identical to the motions that can occur within the dual of that coupling network. Most useful coupling networks, such as mechanisms within machinery, are both overconstrained and underconstrained. Motions of machine parts attributable to underconstraint can be seen thereby making the motions easy to imagine from a drawing. Conversely, internal actions attributable to overconstraint cannot be seen and so are difficult to imagine. Such actions could result in fatigue failure. Thus one possible benefit of creating the dual of a coupling network is that internal actions hidden within it become recognisable motions of bodies in its dual. This article could lead to a better awareness of overconstraint and its dangers.
\end{abstract}

Keywords: action, constraint, freedom, graph, motion

\section{INTRODUCTION}

Two related mathematical concepts are said to be dual if two properties of each are transposed in the other. The Platonic solids provide examples: the number of faces of one of a dual pair of Platonic solids is the number of vertices of the other. Thus, the cube has six faces and eight vertices; its dual, the octahedron, has eight faces and six vertices. It is proposed here that the motion and action screw systems of a coupling network are properties that can be transposed with those of a dual coupling network.

Davies [1] shows how the magnitudes of all actions that can exist within an overconstrained coupling network can be expressed in terms of $C_{\mathrm{N}}$ primary variables, where $C_{\mathrm{N}}$ is the nett degree of constraint (doc) of the coupling network. Also, in reference [1], it is shown how the magnitudes of all possible relative motions of pairs of bodies within an underconstrained coupling network can be expressed in terms of $F_{\mathrm{N}}$ primary variables, where $F_{\mathrm{N}}$ is the nett degree of freedom (dof) of the coupling network.

The symmetry of the dual equations used in reference [1] suggests that a coupling network has a dual coupling network, in which the geometric description of action screws in one is identical to the geometric description of motion screws in the other. The object of this article is to establish a means of finding the dual of a coupling network and to study the dual properties of both. To do this, a starting point is the coupling graph of a coupling network.

\section{DUAL COUPLING GRAPHS}

A geometric graph comprises points called nodes and $e$ lines called edge joining pairs of nodes. For works dedicated to graph theory and networks refer to $[2,3]$. A coupling network $N$ has a coupling graph $G_{\mathrm{C}}$, in which each node of $G_{\mathrm{C}}$ represents a body of $N$ and each edge of $G_{C}$ represents a coupling of $N$. Figure 1 shows a spatial coupling network comprising four bodies and five couplings, which is referred to hereafter in this article as $N^{+}$. It was devised by Baker [4] and has been used as an example on several occasions, most recently in reference [1]. The coupling network has no utility: it is chosen because its action and motion systems are well documented and because the task of finding the dual is a challenging one. Figure 2 shows $G_{\mathrm{C}}^{+}$, the coupling graph of the coupling network $N^{+}$.

\subsection{Spanning tree $T$ of a graph}

Any connected subgraph of a graph $G$ that contains all nodes of $G$ but no circuits is a spanning tree $T$ 


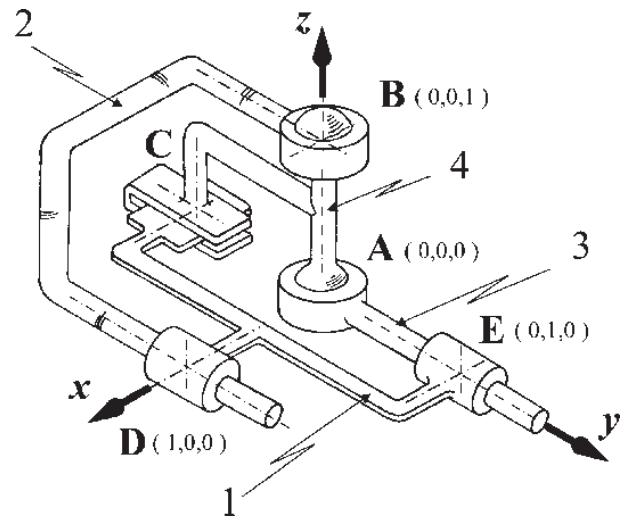

Fig. 1 A spatial coupling network $N^{+}$that is both overconstrained and underconstrained

of $G$. A graph can have several spanning trees. The edges of $G$ that belong to $T$ are called branches and the other edges of $G$ not belonging to $T$ are called chords. The number of independent circuits (loops) $l$ of a graph is the same as the number of chords; the number of independent cutsets $k$ is the same as the number of branches. An independent circuit contains exactly one chord; an independent cutset contains exactly one branch. The three edges shown as thick lines in Fig. 2 are the branches of the chosen tree of the coupling graph $G_{\mathrm{C}}^{+}$and the other two edges are the chords. There are, therefore two independent circuits each containing one chord of the tree and three cutsets each containing one branch. The two independent circuits are assigned a positive sense by the arrows attached to the arcs shown in Fig. 2. Within each arc is a letter that is the label for the circuit and that of the corresponding chord. The labels provided for nodes and edges, and the choice of the tree and the positive senses assigned to edges and circuits, are identical to those used in reference [1]. The cutsets of $G_{\mathrm{C}}^{+}$that were needed in reference [1] are not required in this article.

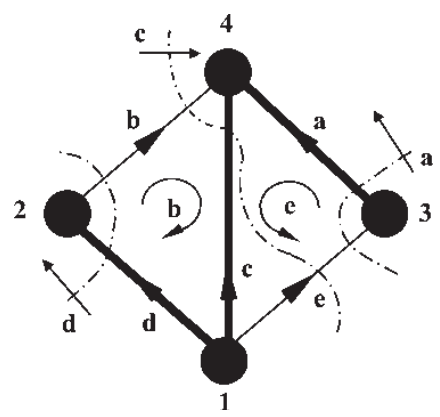

Fig. 2 The coupling graph $G_{\mathrm{C}}^{+}$of the coupling network $N^{+}$shown in Fig. 1 with edges of the chosen tree represented by thick lines; also with nodes numbered and with edges and circuits labelled and assigned positive senses indicated by arrows

\subsection{Planar graphs}

A planar graph is a graph that can be embedded onto the surface of a sphere or plane without having any pairs of edges that cross one another. When a planar graph is embedded onto the surface of a sphere, each area of that spherical surface that is surrounded by edges of the graph is called a region [2] or face [3] of the graph. The graph can be thought of as embedded on a plane provided that the external area is regarded as a region that is no different to any other region as is the case when the graph is embedded on a sphere. The number of regions of a planar graph is one more than $l$, the number of independent circuits. This is because the edges forming the circuit surrounding any one region can be regarded as a linear combination of the other independent circuits. Figure 2 is a planar graph with three regions.

\subsection{Dual graphs}

A planar graph $G^{+}$has a dual planar graph $G^{-}$that is created in the following way. Within each region of $G^{+}$there exists one node of $G^{-}$. Each edge of $G^{+}$is crossed by one edge of $G^{-}$. It is convenient later to refer to a pair of crossing edges, one from each of a pair of dual graphs, as corresponding edges.

The graph $G_{\mathrm{C}}^{-}$, dual with $G_{\mathrm{C}}^{+}$, is created in Fig. 3(a), where edges of $G_{\mathrm{C}}^{-}$are shown as dashed lines. $G_{\mathrm{C}}^{-}$is redrawn in Fig. 3(b) with the two branches of the chosen spanning tree identified by thicker lines and with nodes and edges labelled. The two cutsets are identified by dashed lines and labelled with letters $b$ and $e$ corresponding to the labels of the only branches belonging to the cutsets (label $e$ must not be confused with $e$, the number of edges in a coupling graph and the number of couplings in the corresponding coupling network). Arrows indicate the positive senses assigned to edges and cutsets. As explained previously $[\mathbf{1}]$, the choice of spanning tree and the positive senses assigned can be arbitrary decisions. The decisions resulting in Fig. 3(b) are not arbitrary, however, but are made in order that the cutsets $b$ and $e$ of $G_{\mathrm{C}}^{-}$have the same labels as the circuits of $G_{\mathrm{C}}^{+}$. Furthermore, the choices made for the positive senses assigned to the edges and cutsets of $G_{\mathrm{C}}^{-}$are such that the cutset matrix of $G_{\mathrm{C}}^{-}$is identical to the circuit matrix of $G_{\mathrm{C}}^{+}$.

In a circuit or cutset matrix of a graph $G$ each column represents an edge of $G$ and each row represents a circuit or cutset of $G$. An element of a circuit or cutset matrix is zero, if the edge represented by the column does not belong to the circuit or cutset represented by the row. Otherwise, the element is 1 or -1 depending on whether the positive sense assigned to the circuit or cutset corresponds with 

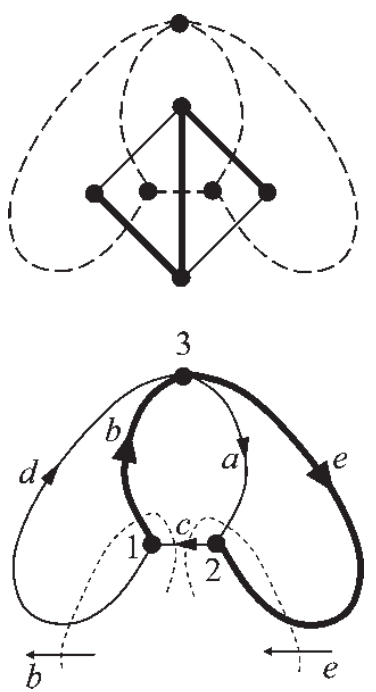

Fig. 3 (a) A reproduction of $G_{\mathrm{C}}^{+}$from Fig. 2 together with its dual graph $G_{\mathrm{C}}^{-}$having edges represented by dashed lines and (b) A reproduction of $G_{\mathrm{C}}^{-}$from Fig. 3(a) with edges of the chosen tree represented by thick lines; also with nodes numbered and with edges and cutsets labelled and assigned positive senses indicated by arrows

the positive sense assigned to the edge. Matrices in this article, as in reference [1], have two external subscripts that indicate the number of rows and columns. The cutset matrix $[\mathbf{Q}]_{k, e}$ of $G_{\mathrm{C}}^{-}$and the circuit matrix $[\mathbf{B}]_{l, e}$ of $G_{\mathrm{C}}^{+}$are

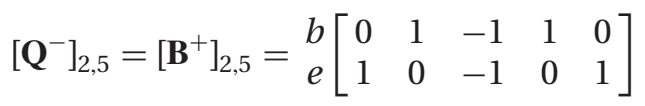

The circuits of $G_{\mathrm{C}}^{-}$are not needed in this article.

\section{DUAL COUPLINGS}

A pair of couplings that exhibit the property that the system of motion screws of one is identical, geometrically, to the system of action screws of the other, and vice versa, will be referred to as dual couplings. The dual of some couplings might be two or more couplings in series or in parallel. For example, the dual of the helical $(\mathrm{H})$ coupling having one dof is what Hunt [5] calls a wrench support having one doc. This is explained, and illustrated by Hunt [5] in his Fig. 12.3. The wrench support comprises a Hooke coupling made from two revolute (R) couplings in series: a helical coupling and a second Hooke coupling with axes aligned parallel with those of the first Hooke coupling. Therefore essentially, the wrench support consists of five couplings in series each allowing one dof.
A cylindrical (C) coupling has a dual that comprises two (C) couplings in series. Such a compound coupling is referred to as a $(\mathrm{C}+\mathrm{C})$ coupling in this article. Two of them can be seen in Fig. 5 labelled $\mathbf{D}$ and $\mathbf{E}$. The arrangement is like a Hooke coupling but made from (C) couplings instead of from revolute $(\mathrm{R})$ couplings. The axial clearances needed to allow translational motion at each of the (C) couplings can be small, because the article is concerned only with instantaneous kinematics. The system of motion screws permitted by a cylindrical coupling is categorized by Hunt [5] as the fifth special 2system of motion screws. See also Phillips [6, 7] on the subject of screw systems. These screws all have axes confined to the same line, but may have any pitch. For the dual $(C+C)$ coupling, the system of action screws that can be transmitted is the same fifth special 2-system of screws. A discussion of the fifth special 4-system of screws that describe the motions allowed by the $(\mathrm{C}+\mathrm{C})$ coupling and the actions that can be transmitted by a single (C) coupling is provided in section 6 .

Other couplings have duals that are single couplings. Hunt [5] illustrates in his Fig. 12.2 what he calls substitute connections for a pair of contacting bodies. All four are possible duals for the revolute (R) coupling, the simplest being the single point contact.

\subsection{Self-dual couplings}

Simplest of all to understand are self-dual couplings. The spherical (S) coupling and its variant, the planar or ebene (E) coupling, provide examples that are evident in the spatial coupling networks shown in Figs 1 and 5. The system of motion screws permitted by a spherical coupling is categorized by Hunt [5] as the second special 3-system of screws; all have the same pitch and the ISAs form a star of $\infty^{2}$ lines in all directions but having one point in common, the centre of the sphere. For a spherical coupling, the pitches of the screws are all zero, so the permitted motions of the bodies coupled by an (S) coupling are all angular velocities about axes passing through the sphere centre. Being a self-dual coupling, the action screws of the (S) coupling are geometrically identical to the motion screws, having the same pitch and the same ISAs. Thus, the actions that can be transmitted by a spherical coupling are all forces with lines of action that pass through the sphere centre.

The system of motion screws permitted by the ebene (E) coupling is categorized by Hunt [5] as the fifth special 3-system of motion screws. This special 3-system comprises screws of infinite pitch anywhere in space that all have a direction parallel to the same plane together with all screws of the same pitch $p$ with ISAs perpendicular to that plane. For 
the (E) coupling, the pitch $p$ is zero. In Figs 1 and 5, the (E) coupling labelled $\mathbf{C}$ has a characteristic plane $z=0$ or any plane parallel to it. The motions are, therefore, translational velocities in any direction parallel with the plane $z=0$ and angular velocities about any axis perpendicular to the plane $z=0$. Being a self-dual coupling, the action screws of the (E) coupling are geometrically identical to the motion screws, having the same pitch and the same ISAs. Thus, the actions that can be transmitted are torque in any direction parallel with the plane $z=0$ and forces along any line of action perpendicular to the plane $z=0$.

\section{DUAL OF A COUPLING NETWORK}

Consider now a coupling network $N^{+}$with a planar coupling graph $G_{\mathrm{C}}^{+}$. There is a second coupling network $N^{-}$that will be said to be dual with $N^{+}$ provided that two conditions are met. First, the coupling graph $G_{\mathrm{C}}^{-}$of $N^{-}$must be the dual graph of $G_{\mathrm{C}}^{+}$. Second, corresponding edges of $G_{\mathrm{C}}^{+}$and $G_{\mathrm{C}}^{-}$ must represent dual couplings. The two steps required to create the dual of a coupling network are represented on the flowchart shown in Fig. 4 by the dashed lines at the top and bottom. The dual coupling networks $N^{+}$and $N^{-}$are represented in the flowchart by boxes bordered by double lines found on the extreme left and right.

For the spatial coupling network $N^{+}$shown in Fig. 1, the dual coupling network $N^{-}$is shown in Fig. 5.

\section{DUAL EQUATIONS FOR DUAL COUPLING NETWORKS}

Attention is now focused on the remainder of the flowchart shown in Fig. 4. The flowchart is in two halves with mirror symmetry about an imaginary vertical line through the centre. The left-hand half summarizes the stages, explained in reference [1], that are necessary to formulate two equations. The starting point is the box that has been referred to, the middle of the three boxes on the far left labelled coupling network $N^{+}$. The two equations can be found, one above the other, at the bottom of the chart, left of centre. These equations provide an analysis of any motions and actions that can exist within the coupling network $N^{+}$. Two equations are

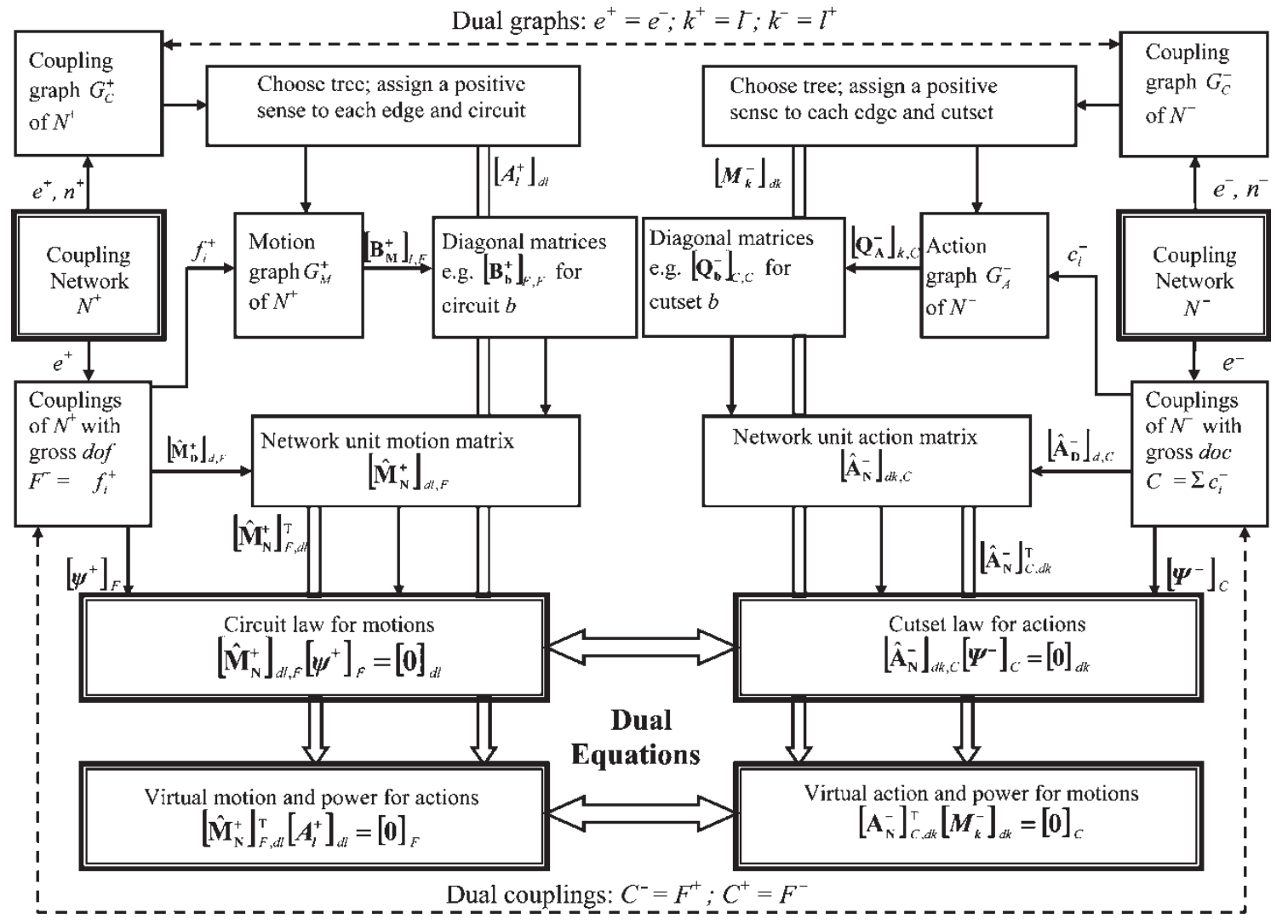

Fig. 4 A flowchart showing progress from dual coupling networks $N^{+}$and $N^{-}$to dual equations that use adaptations of Kirchhoff's laws and virtual power methods for action and motion analyses 


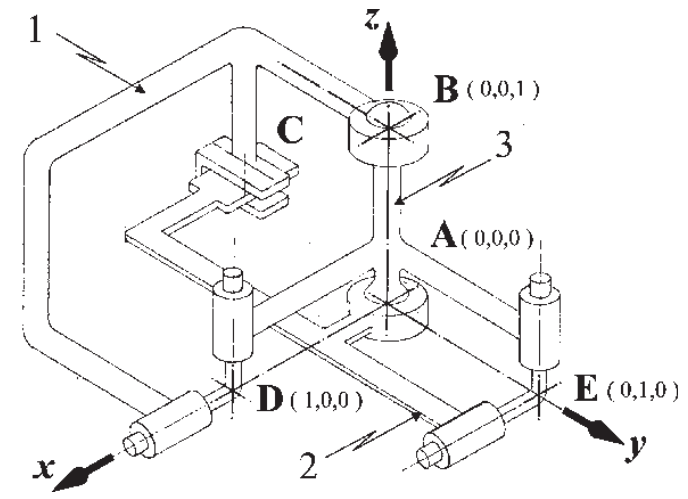

Fig. 5 The coupling network $N^{-}$that is the dual of $N^{+}$ shown in Fig. 1

derived in reference [1] for each task; two for action analysis and two for motion analysis. The reason that only one equation for each task appears in Fig. 4 for $\mathrm{N}^{+}$is that, for $\mathrm{N}^{+}$and most mechanisms, those equations are easier to assemble [1] than the two that are absent.

Both equations in the left-hand half of Fig. 4 require the assembly of the network unit motion matrix $\left[\hat{\mathbf{M}}_{\mathrm{N}}^{+}\right]_{d l, F}$ for $N^{+}$, but neither requires the network unit action matrix. The dimension $d(1 \leqslant d \leqslant$ 6 ) is the order of the system to which all screws under consideration belong. Usually $d$ is six as it is throughout this article and, for the network unit motion matrix, the screws are motion screws. $F$ is the gross dof of a coupling network equal to $\Sigma f$ the sum of the gross dof of all couplings of the network. The network motion matrix itself requires the assembly of the motion graph $G_{\mathrm{M}}^{+}$for $\mathrm{N}^{+}$and its circuit matrix $\left[\mathbf{B}_{\mathrm{M}}^{+}\right]_{l, F}$. The spatial coupling network shown in Fig. 1, and now referred to as $N^{+}$, has been studied in detail previously: all four equations are formulated and solved in reference [1]. The solutions of the two equations of interest are repeated in sections 5.1.1 and 5.1.2 that follow so that comparisons can be made with the dual equations for $N^{-}$.

The right-hand half of the flowchart in Fig. 4 has a role similar to that of the left-hand half. It leads from the box labelled coupling network $N^{-}$to two more equations. Symmetrically with the equations relating to $N^{+}$, these equations can be found, again one above the other and at the bottom of the chart, but to the right of centre. The equations for $N^{-}$are the two equations of the four presented in reference $[\mathbf{1}]$ that are not used for $N^{+}$. The reason for this is that the equations for $N^{-}$are the duals of the equations for $N^{+}$. The solutions of a pair of dual equations describe screw systems that are geometrically identical. Thus, the systems of motion and action screws for $N^{-}$are identical to the systems of action and motion screws, respectively, for $N^{+}$. Because the two equations used for $\mathrm{N}^{+}$are the easiest to assemble of the four available, it follows that the two equations used for the dual coupling network $N^{-}$are also the easiest of the four to assemble for $N^{-}$. Statements dual to those made in the concluding two sentences of the previous paragraph can be made about the right-hand half of the flowchart. Both equations for $N^{-}$require the network unit action matrix $\left[\hat{\mathbf{A}}_{\mathrm{N}}^{-}\right]_{d k, C}$ but neither requires the network unit motion matrix. The network action matrix itself requires the assembly of the action graph $G_{\mathrm{A}}^{-}$for $N^{-}$and its cutset matrix $\left[\mathbf{Q}_{\mathrm{A}}^{-}\right]_{k, C}$ but not the motion graph and its circuit matrix. In the next section, the network unit action matrix for $\mathrm{N}^{-}$is assembled in preparation for the solution of the two equations for $N^{-}$.

\subsection{Action and motion analyses of the coupling network $N^{-}$shown in Fig. 5}

As mentioned earlier, and illustrated in Fig. 4, the two equations for $N^{-}$require the action graph $G_{\mathrm{A}}^{-}$of $N^{-}$ and its cutset matrix $\left[\mathbf{Q}_{\mathrm{A}}^{-}\right]_{k, C}$. It is explained in reference [1] that the action graph $G_{\mathrm{A}}^{-}$is created by replacing each edge of the coupling graph $G_{\mathrm{C}}^{-}$by $c$ edges in parallel, where $c$ is the gross doc of the coupling represented by the edge of $G_{\mathrm{C}}^{-}$that is replaced. Because corresponding edges of $G_{\mathrm{C}}^{+}$and $G_{\mathrm{C}}^{-}$represent dual couplings, the $c$-value of a coupling of $N^{-}$is identical to the $f$-value of the dual coupling of $N^{+}$. For $N^{+}$the sum $F^{+}=\Sigma f^{+}=13, l^{+}=2$, and so $\left[\mathbf{B}_{\mathrm{M}}^{+}\right]_{l, F}$ is $\left[\mathbf{B}_{\mathrm{M}}^{+}\right]_{2,13}$. Dually, for $N^{-}$therefore, $C^{-}=\Sigma c^{-}=13, k^{-}=2$, and $\left[\mathbf{Q}_{\mathrm{A}}^{-}\right]_{k, C}$ is $\left[\mathbf{Q}_{\mathrm{A}}^{-}\right]_{2,13}$. The action graph $G_{\mathrm{A}}^{-}$of $N^{-}$is not drawn in this article because it is easily imagined from an inspection of $G_{\mathrm{C}}^{-}$shown in Fig. 3(b). The five edges of $G_{\mathrm{C}}^{-}$are replaced by multiple edges in parallel to create $G_{\mathrm{A}}^{-}$. The sets of parallel edges numbering 3 , $3,3,2$, and 2, respectively, in the sequence $\mathbf{A}-\mathbf{E}$ are the $c$-values of those couplings. The choices of nomenclature and positive senses made for $G_{\mathrm{C}}^{-}$ result in $\left[\mathbf{Q}_{\mathrm{A}}^{-}\right]_{2,13}$ being identical to the circuit matrix $\left[\mathbf{B}_{\mathrm{M}}^{+}\right]_{2,13}$ of $G_{\mathrm{M}}^{+}$provided in reference $[\mathbf{1}]$. The use of superscript plus and minus in this article to distinguish between a coupling network and its dual results in the coupling matrix $\left[\mathbf{B}_{\mathrm{M}}^{+}\right]_{2,13}$ of $N^{+}$, and the diagonal matrices that follow, having a superscript + , whereas in reference [1], this superscript is absent. Thus

$$
\begin{aligned}
& {\left[\mathbf{Q}_{\mathrm{A}}^{-}\right]_{2,13}=\left[\mathbf{B}_{\mathrm{M}}^{+}\right]_{2,13}=} \\
& e\left[\begin{array}{lll:lll:lll:ll:ll}
0 & 0 & 0 & 1 & 1 & 1 & -1 & -1 & -1 & 1 & 1 & 0 & 0 \\
1 & 1 & 1 & 0 & 0 & 0 & -1 & -1 & -1 & 0 & 0 & 1 & 1
\end{array}\right]
\end{aligned}
$$

where column separators identify the corresponding columns of $\left[\mathbf{Q}^{-}\right]_{2,5}$ and the couplings they represent. It follows that the diagonal matrices $\left[\mathbf{Q}_{\mathrm{b}}^{-}\right]_{13,13}$ and 
$\left[\mathbf{Q}_{\mathrm{e}}^{-}\right]_{13,13}$, with diagonal elements provided by the rows of $\left[\mathbf{Q}_{\mathrm{A}}^{-}\right]_{2,13}$, are identical to $\left[\mathbf{B}_{\mathrm{b}}^{+}\right]_{13,13}$ and $\left[\mathbf{B}_{\mathrm{e}}^{+}\right]_{13,13}$.

The diagonal matrices are needed, in conjunction with the direct coupling unit action matrix $\left[\hat{\mathbf{A}}_{\mathrm{D}}^{-}\right]_{d, C}$, to create the network unit action matrix $\left[\hat{\mathbf{A}}_{\mathrm{N}}^{-}\right]_{d k, C}$. For $N^{-}$, it has been noted that $C^{-}=13$ so $\left[\hat{\mathbf{A}}_{\mathrm{D}}^{-}\right]_{d, C}$ is $\left[\hat{\mathbf{A}}_{\mathrm{D}}^{-}\right]_{6,13}$. The six rows and 13 columns of $\left[\hat{\mathbf{A}}_{\mathrm{D}}^{-}\right]_{6,13}$ are provided, in transposed form, by the 13 rows and six columns in the right-hand columns of Table 1 and hence

$$
\begin{aligned}
& {\left[\hat{\mathbf{A}}_{\mathrm{D}}^{-}\right]_{6,13}=} \\
& \begin{array}{c}
R \\
S \\
T \\
U \\
U \\
W \\
W
\end{array}\left[\begin{array}{ccc:ccc:ccc:cc:cc}
0 & 0 & 0 & 0 & -1 & 0 & 0 & 1 & 0 & 0 & 0 & 0 & 0 \\
0 & 0 & 0 & 0 & 0 & 0 & 0 & 0 & 0 & 1 & 0 & 0 & 0 \\
1 & 0 & 0 & 1 & 0 & 0 & 0 & 0 & 0 & 0 & 0 & 0 & 0 \\
0 & 1 & 0 & 0 & 1 & 0 & 0 & 0 & 0 & 1 & 0 & 1 & 0 \\
0 & 0 & 1 & 0 & 0 & 1 & 1 & 0 & 0 & 0 & 0 & 0 & 0
\end{array}\right]
\end{aligned}
$$

where column dividers separate contributions from the five couplings in the sequence $\mathbf{A}-\mathbf{E}$ from left to right. From equation (5b) of reference [1]

$$
\left[\hat{\mathbf{A}}_{\mathrm{N}}\right]_{d k, C}=\left[\begin{array}{c}
{\left[\hat{\mathbf{A}}_{\mathrm{D}}\right]_{d, C}\left[\mathbf{Q}_{1}\right]_{C, C}} \\
{\left[\hat{\mathbf{A}}_{\mathrm{D}}\right]_{d, C}[\mathbf{Q}]_{C, C}} \\
\cdots \\
{\left[\hat{\mathbf{A}}_{\mathrm{D}}\right]_{d, C}\left[\mathbf{Q}_{\mathbf{k}}\right]_{C, C}}
\end{array}\right]_{d k, C}
$$

For $N^{-}, k^{-}=2$, so only two diagonal matrices are needed. Instead of labelling these with subscripts 1 and 2 as in equation (4), the labels $b$ and $e$ of the two cutsets of $G_{A}^{-}$are used as subscripts instead. So, for $N^{-}$, equation (4) becomes

$$
\begin{aligned}
& {\left[\hat{\mathbf{A}}_{\mathrm{N}}^{-}\right]_{12,13}=\left[\begin{array}{l}
{\left[\hat{\mathbf{A}}_{\mathrm{D}}^{-}\right]_{6,13}\left[\mathbf{Q}_{\mathrm{b}}^{-}\right]_{13,13}} \\
{\left[\hat{\mathbf{A}}_{\mathrm{D}}^{-}\right]_{6,13}\left[\mathbf{Q}_{\mathrm{e}}^{-}\right]_{13,13}}
\end{array}\right]_{12,13}=} \\
& \begin{array}{c}
R \\
S \\
T \\
U
\end{array}\left[\begin{array}{ccc:ccc:ccc:cc:cc}
0 & 0 & 0 & 0 & -1 & 0 & 0 & -1 & 0 & 0 & 0 & 0 & 0 \\
0 & 0 & 0 & 1 & 0 & 0 & 0 & 0 & -1 & 0 & 1 & 0 & 0 \\
0 & 0 & 0 & 0 & 0 & 0 & 0 & 0 & 0 & 1 & 0 & 0 & 0 \\
0 & 0 & 0 & 1 & 0 & 0 & 0 & 0 & 0 & 0 & 0 & 0 & 0
\end{array}\right] \\
& \begin{array}{llll:lll:lll:ll:ll}
V & 0 & 0 & 0 & 0 & 1 & 0 & 0 & 0 & 0 & 1 & 0 & 0 & 0
\end{array} \\
& W \quad\left[\begin{array}{llllllllll|ll|ll} 
& 0 & 0 & 0 & 0 & 1 & -1 & 0 & 0 & 0 & 0 & 0 & 0
\end{array}\right.
\end{aligned}
$$

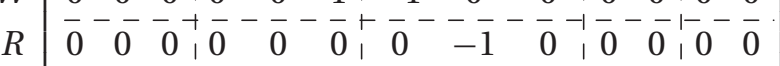

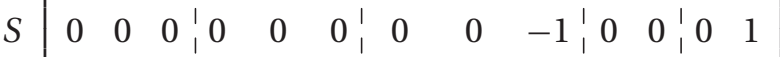

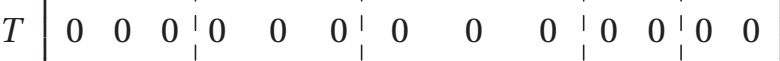

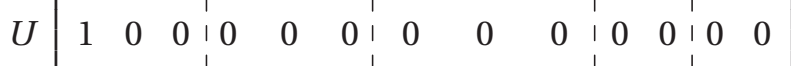

$$
\begin{aligned}
& \left.\begin{array}{llll:lll:lll:ll:ll}
V & 0 & 1 & 0 & 0 & 0 & 0 & 0 & 0 & 0 & 0 & 0 & 1 & 0
\end{array}\right] \\
& W\left[\begin{array}{lll:lll:lll:ll:ll}
0 & 0 & 1 & 0 & 0 & 0 & -1 & 0 & 0 & 0 & 0 & 0 & 0
\end{array}\right]
\end{aligned}
$$

\subsubsection{Action analysis of $N^{-}$using an adaptation of Kirchhoff's cutset law}

This law

$$
\left[\hat{\mathbf{A}}_{\mathrm{N}}\right]_{d k, C}[\boldsymbol{\Psi}]_{C}=[\mathbf{0}]_{d k}
$$

\begin{tabular}{|c|c|c|c|c|}
\hline \multirow[b]{2}{*}{ Coupling label, type, $c$, location } & \multirow[b]{2}{*}{$\begin{array}{l}\text { Force or torque } \\
\text { (direction) }\end{array}$} & \multirow[b]{2}{*}{ Magnitude } & \multicolumn{2}{|c|}{$\begin{array}{l}\text { Unit action screw } \\
\text { coordinates in ray } \\
\text { formation }\end{array}$} \\
\hline & & & $\begin{array}{l}\text { Moment } \\
\text { at origin }\end{array}$ & Force \\
\hline \multirow[t]{3}{*}{ A, spheric, 3 , centre at $(0,0,0)$} & Force $(x)$ & $U_{a}$ & $0,0,0$ & $1,0,0$ \\
\hline & Force $(y)$ & $V_{a}$ & $0,0,0$ & $0,1,0$ \\
\hline & Force $(z)$ & $W_{a}$ & $0,0,0$ & $0,0,1$ \\
\hline \multirow[t]{3}{*}{ B, spheric, 3 , centre at $(0,0,1)$} & Force $(x)$ & $U_{b}$ & $0,1,0$ & $1,0,0$ \\
\hline & Force $(y)$ & $V_{b}$ & $-1,0,0$ & $0,1,0$ \\
\hline & Force $(z)$ & $W_{b}$ & $0,0,0$ & $0,0,1$ \\
\hline \multirow[t]{3}{*}{ C, planar (ebene), 3, plane perpendicular to $z$-axis ${ }^{a}$} & Force $(z)$ & $W_{c}$ & $0,0,0$ & $0,0,1$ \\
\hline & Torque $(x)$ & $R_{c}$ & $1,0,0$ & $0,0,0$ \\
\hline & Torque $(y)$ & $S_{\mathrm{c}}$ & $0,1,0$ & $0,0,0$ \\
\hline \multirow{2}{*}{$\begin{array}{l}\mathbf{D}, \mathrm{C}+\mathrm{C}, 2 \text {, cylinder axes, parallel to } x \text { - and } z \text {-axes, } \\
\text { intersecting at }(1,0,0)\end{array}$} & Force $(y)$ & $V_{d}$ & $0,0,1$ & $0,1,0$ \\
\hline & Torque $(y)$ & $S_{d}$ & $0,1,0$ & $0,0,0$ \\
\hline \multirow{2}{*}{$\begin{array}{l}\text { E, } C+C, 2 \text {, cylinder axes, parallel to } x \text { - and } z \text {-axes, } \\
\text { intersecting at }(0,1,0)\end{array}$} & Force $(y)$ & $V_{e}$ & $0,0,0$ & $0,1,0$ \\
\hline & Torque $(y)$ & $S_{e}$ & $0,1,0$ & $0,0,0$ \\
\hline
\end{tabular}

is listed as equation (6b) in reference $[\mathbf{1}]$. For $N^{-}$, the equation becomes

$$
\left[\hat{\mathbf{A}}_{\mathrm{N}}^{-}\right]_{12,13}\left[\boldsymbol{\Psi}^{-}\right]_{13}=[\mathbf{0}]_{12}
$$

where the vector of 13 unknown action magnitudes,

Table 1 Actions spanning the systems of actions that couplings of the spatial coupling network shown in Fig. 5 can transmit

${ }^{a_{\text {This }}}$ footnote is similar to the one that appears below Table 3 of Appendix 2 in reference [1]. One of the three actions chosen to span this three-system is a force. The line of action of this force can be any line parallel with the $z$-axis. Here, the chosen line is the $z$-axis itself. 
listed in the third column of Table 1, is

$$
\begin{aligned}
& {\left[\boldsymbol{\Psi}^{-}\right]_{13}=} \\
& \quad\left[U_{a}, V_{a}, W_{a}, U_{b}, V_{b}, W_{b}, W_{c}, R_{c}, S_{c}, V_{d}, S_{d}, V_{e}, S_{e}\right]^{\mathrm{T}}
\end{aligned}
$$

It is noted in reference [1] that the rank $\mathrm{m}^{+}$of $\left[\hat{\mathbf{M}}_{\mathrm{N}}^{+}\right]_{12,13}$ is 10 because two of the 12 rows are redundant confirming that $C_{N}^{+}=2$ for $N^{+}$. Dually, the rank $a^{-}$of $\left[\hat{\mathbf{A}}_{\mathrm{N}}^{-}\right]_{12,13}$ is also 10 and so $F_{N}^{-}=2$ for $N^{-}$. Row 9 is redundant because it is empty. Also, the sum of rows 1 and 5 equals the sum of rows 3 and 7 and so any one of these four rows is redundant. When two redundant rows of $\left[\hat{\mathbf{A}}_{N}^{-}\right]_{12,13}$ are removed, the 13 unknowns must satisfy the remaining ten independent equations, hence $C_{N}^{-}=3$ and three primary variables can be chosen.

The solution identifies those of the 13 unknowns that are zero and expresses all others in terms of the three primary variables. Using $V_{a}, W_{a}$, and $S_{c}$ as primary variables, the solution is

$$
\begin{aligned}
& {\left[\boldsymbol{\Psi}^{-}\right]_{13}=} \\
& \quad\left[0, V_{a}, W_{a}, 0,0, W_{a}, W_{a}, 0, S_{c}, 0, S_{c},-V_{a}, S_{c}\right]^{\mathrm{T}}
\end{aligned}
$$

When the unit action coordinates in $\left[\hat{\mathbf{A}}_{\mathrm{D}}^{-}\right]_{6,13}$ are multiplied by the elements of $\left[\boldsymbol{\Psi}^{-}\right]_{13}$, the action matrix thereby created is $\left[\mathbf{A}^{-}\right]_{6,13}$, wherein each of the 13 columns contains six elements that are either all zero or they represent a single action screw that can be transmitted by the coupling. In the condensed version $\left[\mathbf{A}^{-}\right]_{6,5}$, columns of $\left[\mathbf{A}^{-}\right]_{6,13}$ that pertain to the same coupling are added so that each column of $\left[\mathbf{A}^{-}\right]_{6,5}$ represents the system of all action screws that can be transmitted by one of the five couplings. For $N^{-}$

$$
\left[\mathbf{A}^{-}\right]_{6,5}=\left[\begin{array}{ccccc}
0 & 0 & 0 & 0 & 0 \\
0 & 0 & S_{c} & S_{c} & S_{c} \\
0 & 0 & 0 & 0 & 0 \\
0 & 0 & 0 & 0 & 0 \\
V_{a} & 0 & 0 & 0 & -V_{a} \\
W_{a} & W_{a} & W_{a} & 0 & 0
\end{array}\right]
$$

with columns corresponding to couplings in the sequence $\mathbf{A}-\mathbf{E}$. $\left[\mathbf{A}^{-}\right]_{6,5}$ is the same matrix as the one that would have been produced using virtual motions and circuit actions in a virtual power equation but that process would have been lengthier.

This result is now compared with the results of the use of the adaptation of Kirchhoff's circuit law for the motion analysis of $\mathrm{N}^{+}$in reference [1]. For $\mathrm{N}^{+}$, the condensed version $\left[\mathbf{M}^{+}\right]_{d, e}$ of the motion matrix
$\left[M^{+}\right]_{d, F}$ is

$$
\left[\mathbf{M}^{+}\right]_{6,5}=\left[\begin{array}{ccccc}
0 & 0 & 0 & 0 & 0 \\
s_{a} & 0 & 0 & 0 & -s_{a} \\
t_{a} & t_{a} & t_{a} & 0 & 0 \\
0 & 0 & 0 & 0 & 0 \\
0 & 0 & v_{c} & v_{c} & v_{c} \\
0 & 0 & 0 & 0 & 0
\end{array}\right]
$$

$\left[\mathrm{M}^{+}\right]_{6,5}$ is also derived in reference [1] using virtual actions in a virtual power equation, but that process is lengthier.

It is not immediately obvious that $\left[\mathbf{A}^{-}\right]_{6,5}$ and $\left[\mathbf{M}^{+}\right]_{6,5}$ represent the same geometric screw systems. One reason is that the notation used is for action screws in the first matrix and for motion screws in the second. The second reason is that action screws are expressed in ray formation, whereas motion screws are in axis formation. By transposing the first three rows with the last three rows of either matrix, all screws become expressed in the same formation.

\subsubsection{Motion analysis of $N^{-}$using virtual actions and cutset motions in a virtual power equation}

This virtual power equation

$$
\left[\hat{\mathbf{A}}_{\mathrm{N}}^{\mathrm{T}}\right]_{C, d k}\left[\boldsymbol{M}_{k}\right]_{d k}=[\mathbf{0}]_{C}
$$

is listed as equation (11a) in reference [1]. The matrix $\left[\hat{\mathbf{A}}_{\mathrm{N}}^{\mathrm{T}}\right]_{C, d k}$ is the transpose of the network unit action matrix provided above in equation (4). For $N^{-}$, the equation becomes

$$
\left[\hat{\mathbf{A}}_{\mathrm{N}}^{-\mathrm{T}}\right]_{13,12}\left[\boldsymbol{M}_{k}^{-}\right]_{12}=[\mathbf{0}]_{13}
$$

where the vector of 12 unknown cutset motion magnitudes for cutsets $b$ and $e$ is

$$
\begin{aligned}
& \quad\left[\boldsymbol{M}_{k}^{-}\right]_{12}=\left[\left[\boldsymbol{M}_{b}^{-}\right]_{6} \vdots\left[\boldsymbol{M}_{e}^{-}\right]_{6}\right]^{\mathrm{T}}= \\
& \quad\left[r_{b}, s_{b}, t_{b}, u_{b}, v_{b}, w_{b}, r_{e}, s_{e}, t_{e}, u_{e}, v_{e}, w_{e}\right]^{\mathrm{T}}
\end{aligned}
$$

Because the rank $a^{-}$of $\left[\hat{\mathbf{A}}_{\mathrm{N}}^{-}\right]_{12,13}$ and that of its transpose is 10 , three of the 13 rows of its transpose are redundant thereby providing further confirmation that $C_{\mathrm{N}}^{-}=3$. To recognize redundant rows in the transpose, it is sufficient to examine the columns of $\left[\hat{\mathbf{A}}_{\mathrm{N}}^{-}\right]_{12,13}$. It can be seen that column 2 is identical to column 12; the sum of columns 3, 6, and 7 are zero and the sum of columns 9,11 , and 13 are also zero. When three redundant rows of $\left[\hat{\mathbf{A}}_{\mathrm{N}}^{-\mathrm{T}}\right]_{13,12}$ are removed, the 12 unknowns must satisfy the remaining ten independent equations hence $F_{\mathrm{N}}^{-}=2$ and two 
primary variables can be chosen. The redundant rows represent actions attributable to overconstraint that could be present in $N^{-}$; the other ten rows represent virtual actions that could not exist and therefore cannot expend power on the cutset motions. The solution identifies those of the 12 unknowns that are zero and expresses all others in terms of the two primary variables. Using $r_{b}$ and $t_{e}$ as primary variables, the solution is

$$
\left[\boldsymbol{M}_{k}^{-}\right]_{12}=\left[r_{b}, 0,-r_{b}, 0, r_{b}, 0,-r_{b}, 0, t_{e}, 0,0,0\right]^{\mathrm{T}}
$$

This vector can be rearranged as the matrix of cutset motions

$$
\left[\mathbf{M}_{\mathrm{k}}^{-}\right]_{6,2}=\left[\begin{array}{cc}
r_{b} & -r_{b} \\
0 & 0 \\
-r_{b} & t_{e} \\
0 & 0 \\
r_{b} & 0 \\
0 & 0
\end{array}\right]
$$

The columns of $\left[\mathbf{M}_{\mathrm{k}}^{-}\right]_{6,2}$ contain the components of the motion systems for cutsets $b$ and $e$ and therefore also for the pairs of bodies $(1,3)$ and $(2,3)$ directly coupled by couplings $\mathbf{B}$ and $\mathbf{E}$ represented by the branches $b$ and $e$ of $G_{\mathrm{C}}^{-}$. The components of the nett motion systems for all five couplings, after assembly into the network $N$, are obtained by postmultiplying $\left[\mathbf{M}_{\mathrm{k}}^{-}\right]_{6,2}$ by $\left[\mathbf{Q}^{-}\right]_{2,5}$, the cutset matrix of the coupling graph $G_{\mathrm{C}}^{-}$, to give

$$
\begin{aligned}
{\left[\mathbf{M}^{-}\right]_{6,5} } & =\left[\mathbf{M}_{\mathrm{k}}^{-}\right]_{6,2}\left[\mathbf{Q}^{-}\right]_{2,5}= \\
& {\left[\begin{array}{ccccc}
-r_{b} & r_{b} & 0 & r_{b} & -r_{b} \\
0 & 0 & 0 & 0 & 0 \\
t_{e} & -r_{b} & t_{c} & -r_{b} & t_{e} \\
0 & 0 & 0 & 0 & 0 \\
0 & r_{b} & -r_{b} & r_{b} & 0 \\
0 & 0 & 0 & 0 & 0
\end{array}\right] }
\end{aligned}
$$

where $t_{c}=r_{b}-t_{e} .\left[\mathbf{M}^{-}\right]_{6,5}$ is the same matrix as the one that would have been produced using the adaptation of Kirchhoff's circuit law, but that process would have been lengthier.

This result is now compared with the results of a virtual power equation using virtual motions and circuit actions for the action analysis of $N^{+}$in reference [1]. For $N^{+}$, the circuit action matrix $\left[\mathbf{A}_{1}^{+}\right]_{d, l}$, derived from the vector $\left[A_{l}^{+}\right]_{d l}$, is

$$
\left[\mathbf{A}_{1}^{+}\right]_{6,2}=\left[\begin{array}{cc}
0 & 0 \\
U_{b} & 0 \\
0 & 0 \\
U_{b} & -U_{b} \\
0 & 0 \\
-U_{b} & W_{e}
\end{array}\right]
$$

The columns of $\left[\mathbf{A}_{1}^{+}\right]_{6,2}$ contain the components of the action systems for circuits $b$ and $e$ and therefore also for the couplings $\mathbf{B}$ and $\mathbf{E}$ represented by the chords $b$ and $e$ of $G_{\mathrm{C}}^{+}$. To obtain the components of action systems for all five direct couplings of $N^{+}$ $\left[\mathbf{A}_{1}^{+}\right]_{6,2}$ can be postmultiplied by $\left[\mathbf{B}^{+}\right]_{2,5}$, the circuit matrix of the coupling graph $G_{\mathrm{C}}^{+}$, to give

$$
\begin{aligned}
{\left[\mathbf{A}^{+}\right]_{6,5} } & =\left[\mathbf{A}_{1}^{+}\right]_{6,2}\left[\mathbf{B}^{+}\right]_{2,5}= \\
& {\left[\begin{array}{ccccc}
0 & 0 & 0 & 0 & 0 \\
0 & U_{b} & -U_{b} & U_{b} & 0 \\
0 & 0 & 0 & 0 & 0 \\
-U_{b} & U_{b} & 0 & U_{b} & -U_{b} \\
0 & 0 & 0 & 0 & 0 \\
W_{e} & -U_{b} & W_{c} & -U_{b} & W_{e}
\end{array}\right] }
\end{aligned}
$$

where $W_{c}=U_{b}-W_{e} \cdot\left[\mathbf{A}^{+}\right]_{6,5}$ is also produced in reference [1] using the adaptation of Kirchhoff's cutset law but that process is lengthier.

\section{DISCUSSION}

In this section, the results are re-examined in a new way and the instantaneous kinematics of the $(\mathrm{C}+\mathrm{C})$ coupling is explained.

\subsection{Results from adaptations of Kirchhoff's laws (section 5.1.1)}

Adaptations of Kirchhoff's laws do not involve cutset motions and circuit actions. It is recognised in reference [1] that these concepts provide the most compact record of the motions allowed and actions that can be present in a coupling network. An interesting matter for discussion arises by presenting the results of section 5.1.1 in terms of cutset motions and circuit actions. In reference $[\mathbf{1}]$, the virtual power method involving cutset motions and virtual actions is used for the motion analysis of $N^{+}$. The choice of spanning tree in reference [1], used again in this article for $G_{\mathrm{C}}^{+}$, results in two of the three cutset motion screw systems being 2-systems requiring two independent motions to span them. For both dual coupling graphs $G_{\mathrm{C}}^{+}$and $G_{\mathrm{C}}^{-}$, there are eight possible spanning trees. For $G_{\mathrm{C}}^{+}$, these are listed in 
reference [3] (See page 46, exercise 9.4, and page 154 for the answer). Each spanning tree has its unique set of branches and chords with unique sets of circuits and cutsets corresponding to them. Regardless of which of the eight trees is chosen, at least one screw system is of order two. This is not inevitable as an inspection of a Fig. 1 reveals. There is another choice of cutsets for $\mathrm{N}^{+}$, which produces simpler results. If the three chosen cutsets for $N^{+}$are those that isolate nodes 1,3 , and 4, respectively, each motion system is a 1-system as the central column of Table 2 records. However, these cutsets, having edges listed in the left-hand column of Table 2, do not correspond to a spanning tree of $G_{\mathrm{C}}^{+}$.

Listed in the right-hand column of Table 2 are the sets of edges of $G_{\mathrm{C}}^{-}$corresponding to the edges of $G_{\mathrm{C}}^{+}$ listed in the left-hand column. The edges of these sets form circuits of $G_{\mathrm{C}}^{-}$, but not circuits that correspond to a spanning tree of $G_{\mathrm{C}}^{-}$. Note that circuit CDE is independent of the other two, but circumscribes the outer region of $G_{\mathrm{C}}^{-}$when that graph is represented geometrically in the manner shown in Fig. 3. With this choice of circuits, the circuit actions of $N^{-}$and the cutset motions of $N^{+}$are geometrically identical; only the units differ.

\subsection{Results from virtual work equations (section 5.1.2)}

For circuit actions in $N^{+}$and cutset motions in $N^{-}$, there is no way of avoiding a 2-system of screws. In Table 3, the circuits of $N^{+}$and the cutsets of $N^{-}$ are those associated with the tree originally chosen in reference $[\mathbf{1}]$.

\subsection{Motion at the $(C+C)$ coupling labelled $E$ in Fig. 5}

A cylindrical (C) coupling allows motions based on screws belonging to a system categorized by Hunt [5] as a fifth special 2-system of motion screws. These screws all share the same ISA, but contain screws of every pitch. For the (C) coupling in $N^{+}$ labelled $\mathbf{E}$ in Fig. 1 this ISA coincides with the $y$-axis, so the motions are twists about the $y$-axis. Although the centre of this $(\mathrm{C})$ coupling is shown to be located at $(0,1,0)$ in Fig. 1, these coordinates are provided only to add clarity. The function of the coupling is unaltered wherever it is located provided that the centre-line of the cylinder lies on the $y$-axis. Reciprocal to this fifth special 2-system of motion screws is a fifth special 4-system of action screws comprising screws of every finite pitch with ISAs that belong to a single infinity of parallel planar pencils of lines. The planar pencils are arranged with their central points on a single straight line and this line is normal to the planes of the pencils. In addition, there are screws of infinite pitch with directions parallel to the ISAs. For the (C) coupling in $N^{+}$ labelled $\mathbf{E}$ in Fig. 1, the line of central points of the planar pencils is the $y$-axis.

A $(C+C)$ coupling, being the dual of a single $(C)$ coupling, can transmit actions based on screws belonging to a fifth special 2-system of action screws. For the $(\mathrm{C}+\mathrm{C})$ coupling in $N^{-}$labelled $\mathbf{E}$ in Fig. 5, the only ISA coincides with the $y$-axis so the actions are wrenches of any pitch with a line of action on the $y$-axis. Like the $(\mathrm{C})$ coupling in Fig. 1, the location $(0,1,0)$ of the corner of the L-shaped intermediate body linking the two $(\mathrm{C})$ couplings that comprise the $(\mathrm{C}+\mathrm{C})$ coupling is of no importance except that it must lie on the $y$-axis. It is the motions that occur at the $(\mathrm{C}+\mathrm{C})$ coupling that are of interest and they are affected by the location of $\mathbf{E}$ on the $y$-axis.

Couplings $\mathbf{A}$ and $\mathbf{E}$ are represented in $G_{\mathrm{C}}^{-}$by edges belonging to cutset $e$ and no other cutset. The motion system for cutset $e$ is a 2-system spanned by angular velocity vectors of magnitude $-r_{b}$ and $t_{e}$ having rotation axes along the $x$ - and $z$-axes, respectively. Cutset $e$ isolates member 2 from all others. The $\infty^{1}$ resultant motions can be rotations about any axis among the lines belonging to the pencil of $\infty^{1}$ lines in the plane $y=0$ having the origin, the centre of coupling A, as the common point. This 2-system is, as it must be, a subsystem of the 4 -system of motions allowed by the $(\mathrm{C}+\mathrm{C})$ coupling at $\mathbf{E}$.

For the $(C+C)$ coupling labelled $\mathbf{E}$ in Fig. 5, the motions that occurs at each of the two $(\mathrm{C})$ couplings

Table 2 Summary of results from section 5.1.1

\begin{tabular}{|c|c|c|c|c|}
\hline \multicolumn{2}{|c|}{ Coupling network $N^{+}$} & \multirow[b]{2}{*}{$\begin{array}{l}\text { Screw system as } \\
\text { classed by Hunt [5] }\end{array}$} & \multicolumn{2}{|c|}{ Coupling network $N^{-}$} \\
\hline $\begin{array}{l}\text { Cutset of } \\
\text { couplings }\end{array}$ & $\begin{array}{l}\text { Motion(s) spanning the } \\
\text { cutset motion systems }\end{array}$ & & $\begin{array}{l}\text { Action(s) spanning the } \\
\text { circuit action systems }\end{array}$ & $\begin{array}{l}\text { Circuit of } \\
\text { couplings }\end{array}$ \\
\hline CDE & $\begin{array}{l}\text { Translational velocity } \\
v_{c} \text { parallel with the } \\
y \text {-axis }\end{array}$ & $\begin{array}{l}\text { First (and lone) } \\
\text { special 1-system }\end{array}$ & $\begin{array}{l}\text { Torque } S_{c} \text { parallel with } \\
\text { the } y \text {-axis }\end{array}$ & CDE \\
\hline $\mathbf{A E}$ & $\begin{array}{l}\text { Angular velocity } \\
s_{a} \text { about the } y \text {-axis }\end{array}$ & 1-system (zero pitch) & $\begin{array}{l}\text { Force } V_{a} \text { along the } \\
y \text {-axis }\end{array}$ & $\mathbf{A E}$ \\
\hline ABC & $\begin{array}{l}\text { Angular velocity } \\
t_{a} \text { about the } z \text {-axis }\end{array}$ & 1-system (zero pitch) & $\begin{array}{l}\text { Force } W_{a} \text { along the } \\
z \text {-axis }\end{array}$ & ABC \\
\hline
\end{tabular}


Table 3 Summary of results from section 5.1.2

\begin{tabular}{|c|c|c|c|c|}
\hline \multicolumn{2}{|c|}{ Coupling network $N^{+}$} & \multirow[b]{2}{*}{$\begin{array}{l}\text { Screw system as } \\
\text { classed by Hunt [5] }\end{array}$} & \multicolumn{2}{|c|}{ Coupling network $N^{-}$} \\
\hline $\begin{array}{l}\text { Circuit of } \\
\text { couplings }\end{array}$ & $\begin{array}{l}\text { Action(s) spanning the } \\
\text { circuit action systems }\end{array}$ & & $\begin{array}{l}\text { Motion(s) spanning the cutset } \\
\text { motion systems }\end{array}$ & $\begin{array}{l}\text { Cutset of } \\
\text { couplings }\end{array}$ \\
\hline BCD & $\begin{array}{l}\text { Force } U_{b} \text { along the line } \\
\text { through }(1,0,0) \text { and } \\
(0,0,1)\end{array}$ & 1-system (zero pitch) & $\begin{array}{l}\text { Angular velocity } r_{b} \text { about the } \\
\text { line through }(1,0,0) \text { and } \\
(0,0,1)\end{array}$ & BCD \\
\hline ACE & $\begin{array}{l}\text { Force } U_{b} \text { along the } \\
x \text {-axis and force } W_{e} \\
\text { along the } z \text {-axis }\end{array}$ & $\begin{array}{l}\text { First special 2-system } \\
\quad \text { (zero pitch) }\end{array}$ & $\begin{array}{l}\text { Angular velocity } r_{b} \text { about the } \\
x \text {-axis and angular } \\
\text { velocity } t_{e} \text { about the } z \text {-axis }\end{array}$ & ACE \\
\hline
\end{tabular}

are now examined separately. The (C) coupling that has a centre-line parallel with the $x$-axis experiences angular velocity of magnitude $-r_{b}$ about the centreline together with a translational velocity parallel with that centre-line. This translational velocity is $t_{e}$ multiplied by $(-1)$, the negative of the $y$-coordinate at $\mathbf{E}$. Together these two motions represent a twist rate having angular velocity magnitude $-r_{b}$ and pitch $p_{x}=(-1) t_{e} /\left(-r_{b}\right)=t_{e} / r_{b}$. The other (C) coupling at $\mathbf{E}$, the one parallel with the $z$-axis, experiences angular velocity of magnitude $t_{e}$ about the centre-line also with a translational velocity parallel with that centre-line. This translational velocity is $-r_{b}$ multiplied by (1), the $y$-coordinate at $\mathbf{E}$. Together these two motions represent a twist rate having angular velocity magnitude $t_{e}$ and pitch $p_{z}=\left(-r_{b}\right) / t_{e}$.

To allow translation to occur along the arms of the L-shaped intermediate body linking, the two (C) couplings there must be some space, evident in Fig. 5, which is not needed in a conventional Hooke coupling. The translation can be infinitesimal however because motion need be no more than transitory to be regarded as motion. This leads to the third conclusion in the section that follows.

\section{CONCLUSIONS}

1. For a coupling network that has a planar coupling graph, a procedure is explained whereby another coupling network is created such that the two have dual properties described below. Such pairs are called dual coupling networks.

2. In reference [1] two sets of dual equations are presented for the analysis of actions that can exist and motions that are permitted within coupling networks. It is demonstrated here that pairs of dual equations are identical for dual coupling networks. This means that the geometrical description of actions that can exist within one of a dual pair of coupling networks is identical to description of the motions that are permitted within the other.

3 . The methods described in reference [1], and in this article, are insufficient on their own to distinguish between a transitory nett dof and the freedom to perform a finite displacement. Hunt [5] discusses the distinction in some detail.

4. Regardless of whether the freedom is transitory, the duality described in this article is instantaneous. Generally, the dual of an underconstrained coupling network is altered by any displacement of the former. Some gear trains provide exceptions to this generalization.

5. One difficult challenge is to recognize that the dual of a cylindrical (C) coupling is a coupling comprising two cylindrical couplings in series called here the $(\mathrm{C}+\mathrm{C})$ coupling.

6. Evidence for the possible benefit mentioned in the abstract cannot be found in Table 3 . The motions of bodies within $N^{-}$, recorded in the right-hand columns of Table 3 , are not easily imagined without model making. This is contrary to evidence provided by simpler coupling networks. It is suggested that a reason for this difficulty is unfamiliarity with the $(\mathrm{C}+\mathrm{C})$ coupling and the motions it allows.

7. Contrastingly, the motions of bodies within $N^{+}$ are easy to imagine. These are described in reference [1] and, in this article, in the left-hand columns of Table 2. Thus, if the task had been to find the actions that can exist in $N^{-}$, instead of $N^{+}$, then those actions, described in the righthand columns of Table 2, could have been found from an inspection of the dual $N^{+}$without recourse to analysis of the kind provided in reference $[\mathbf{1}]$.

8. Much is written about kinematics in machinery: motions that could occur as a consequence of underconstraint. The article suggests that there is as much that can be written about internal actions that could exist as a consequence of overconstraint.

\section{REFERENCES}

1 Davies, T. H. Freedom and constraint in coupling networks. Proc. IMechE, Part C: J. Mechanical Engineering Science, 2006, 220(C7), 989-1010. 
2 Busacker, R. G. and Saaty, T. L. Finite graphs and networks: an introduction with applications, 1965 (McGraw-Hill, New York).

3 Wilson, R. J. Introduction to graph theory, 4th edition, 1996 (Longman, Harlow, Essex, England).

4 Baker, J. E. On relative freedom between links in kinematic chains with cross-jointing. Mech. Mach. Theory, 1980, 15(5), 397-413.

5 Hunt, K. H. Kinematic geometry of mechanisms. The Oxford engineering science series, 7, 1990 (Oxford University Press, Oxford).

6 Phillips, J. Freedom in machinery: introducing screw theory, Vol. 1, 1984 (Cambridge University Press, Cambridge).

7 Phillips, J. Freedom in machinery: screw theory exemplified, Vol. 2, 1990 (Cambridge University Press, Cambridge).

\section{APPENDIX}

\section{Notation}

$a$

$[\mathbf{A}]_{d, C}$

$[\mathbf{A}]_{d, e}$

$\left[\mathbf{A}_{\mathbf{D}}\right]_{d, C},\left[\hat{\mathbf{A}}_{\mathbf{D}}\right]_{d, C}$

$\left[A_{l}\right]_{d l}$

$\left[\mathbf{A}_{l}\right]_{d, l}$

$\left[\hat{\mathbf{A}}_{\mathbf{N}}\right]_{d k, C}$

$[\mathbf{B}]_{l, e}$

$\left[\mathbf{B}_{\mathbf{b}}\right]_{F, F}$

$\left[\mathbf{B}_{\mathbf{M}}\right]_{1, F}$

$c$

C

$C_{\mathrm{N}}$ rank of the network unit action $\operatorname{matrix}\left[\hat{\mathbf{A}}_{\mathbf{N}}\right]_{d k, C}$

action matrix of a coupling network in terms of $C_{N}$ primary variables

a condensed version of $[\mathbf{A}]_{d, C}$ with one column for each coupling

action and unit action matrices

of the direct couplings of a coupling network

$d l$ action system components for all $l$ circuits

circuit action matrix

network unit action matrix of a coupling network

circuit matrix of the coupling graph $G_{\mathrm{C}}$

diagonal matrix with diagonal elements corresponding to the row of $\left[\mathbf{B}_{\mathbf{M}}\right]_{l, F}$ representative of circuit $b$ (and similarly for circuit $e$ )

circuit matrix of motion graph $G_{M}$

the gross degree of constraint

(doc) of a direct coupling

the gross doc of a coupling

network $=\Sigma c$

the nett doc of a coupling

network $d$

$e$

$f$

$F$

$F_{\mathrm{N}}$

$k$

$l$

$m$

$\left[\boldsymbol{M}_{b}\right]_{d}$

$[\mathbf{M}]_{d, F}$

$[\mathbf{M}]_{d, e}$

$\left[\boldsymbol{M}_{k}\right]_{d k}$

$\left[\mathbf{M}_{k}\right]_{d, k}$

$\left[\hat{\mathbf{M}}_{N}\right]_{d l, F}$

$p$

$[\mathbf{Q}]_{k, e}$

$\left[\mathbf{Q}_{A}\right]_{k, \mathrm{C}}$

$\left[\mathbf{Q}_{b}\right]_{C, C}$

$(r, s, t ; u, v, w)$

$(R, S, T ; U, V, W)$

$\omega$

$[\boldsymbol{\psi}]_{C}$ dimension $(1 \leqslant d \leqslant 6)$; the order of the system to which all screws under consideration (motion or action screws) belong; usually $d$ is six as it is throughout this article the number of couplings in a coupling network and edges of $G_{C}$

the gross degree of freedom (dof) of a direct coupling

the gross dof of a coupling network $=\Sigma f$

the nett dof of a coupling network the number of cutsets of graphs $G_{C}$ and $G_{A}$

the number of circuits (loops) of graphs $G_{C}$ and $G_{M}$

the rank of the network unit motion matrix

$d$ motion system components for cutset $b$ (similarly for cutset $e$ )

motion matrix of a coupling network in terms of $F_{N}$ primary variables

a condensed version of $[\mathbf{M}]_{d, F}$ with one column for each coupling

$d k$ motion system components

for all $k$ cutsets

cutset motion matrix

network unit motion matrix of a coupling network

the pitch of a screw

cutset matrix of the coupling

graph $G_{\mathrm{C}}$

cutset matrix of action

graph $G_{\mathrm{A}}$

diagonal matrix with diagonal

elements corresponding to the row of $\left[\mathbf{Q}_{A}\right]_{k, C}$ representative of cutset $b$ (and similarly for cutset $e$ )

motion coordinates in axis formation

action coordinates in ray formation

angular velocity magnitude $C$ generalized action magnitudes (force or torque) 